https://doi.org/10.15407/ujpe64.7.631

A. RYMBEKOVA

Joint Institute for Nuclear Research

(6, Joliot-Curie Str, Dubna, Moscow Region, Russia; e-mail: aierke@jinr.ru)

\title{
STUDY OF THE POLARIZED GLUON STRUCTURE OF A PROTON VIA PROMPT-PHOTON PRODUCTION IN THE SPD EXPERIMENT AT THE NICA COLLIDER
}

\begin{abstract}
Photons produced in the hard scattering of partons, named prompt photons, provide information about the internal structure of hadrons. The NICA collider has the possibility to provide new data to study the production of prompt photons in non-polarized and polarized protonproton collisions, which gives an access to spin-dependent parton distribution functions for gluons. Unpolarized and polarized physics with prompt photons and capabilities of the SPD detector in such measurements is discussed.

Keywords: polarized structure of a nucleon, prompt photons, gluons, SPD.
\end{abstract}

\section{Prompt Photons}

Prompt photons are photons produced in the hard scattering of partons. According to the factorization theorem, the inclusive cross-section for the production of a prompt photon in collisions of $h_{A}$ and $h_{B}$ hadrons can be written as follows:

$$
\begin{aligned}
& d \sigma_{A B}=\sum_{a, b=q, \bar{q}, g} \int d x_{a} d x_{b} f_{a}^{A}\left(x_{a}, Q^{2}\right) f_{b}^{B}\left(x_{b}, Q^{2}\right) \times \\
& \times d \sigma_{a b \rightarrow \gamma X}\left(x_{a}, x_{b}, Q^{2}\right) .
\end{aligned}
$$

The function $f_{a}^{A}\left(f_{b}^{B}\right)$ is the parton density for $h_{A}$ $\left(h_{B}\right)$ hadron, $x_{a}\left(x_{b}\right)$ is the fraction of the momentum of $h_{A}\left(h_{B}\right)$ hadron carried by parton $a(b)$, and $Q^{2}$ is the square of the 4-momentum transferred in the hard scattering process, and $\sigma_{a b \rightarrow \gamma X}\left(x_{a}, x_{b}, Q^{2}\right)$ represents the cross-section for the hard scattering of partons $a$ and $b[1]$.

The prompt-photon production in hadron collisions is the most direct way to access the gluon structure of hadrons. There are two main hard processes for the production of prompt photons: 1) gluon Compton scattering, $g q(\bar{q}) \rightarrow \gamma q(\bar{q})$, which prevails, and 2) quark-antiquark annihilation, $q \bar{q} \rightarrow \gamma g$.

Unpolarized measurements of the differential crosssection of the prompt-photon production in protonproton(antiproton) collisions have already been performed by the fixed-target and collider experiments.

(C) A. RYMBEKOVA, 2019

ISSN 2071-0194. Ukr. J. Phys. 2019. Vol. 64, No. 7
Figure 1 shows the ratio of the measured cross-sections to one predicted by theory as a function of $x_{T}=$ $=2 p_{T} / \sqrt{s}$ [2]. One can see that, for the fixed-target results corresponding to $\sqrt{s} \sim 20 \mathrm{GeV}$, there is a significant disagreement with theoretical expectations that is absent for the high-energy collider results. A new precise measurement could clarify the problem.

\section{Spin Asymmetries}

A measurement of the single transverse spin asymmetry $A_{\gamma}^{N}=\frac{\sigma^{\uparrow}-\sigma^{\downarrow}}{\sigma^{\uparrow}+\sigma^{\downarrow}}$ in the prompt-photon production at high $p_{T}$ in polarized $p$ - $p$ and $d$ - $d$ collisions could provide information about the gluon Sivers function which is mostly unknown at the moment. The numerator of $A_{\gamma}^{N}$ can be expressed as [3]

$\sigma^{\uparrow}-\sigma^{\downarrow}=$

$=\sum_{i} \int^{1} d x_{a} \int d^{2} \mathbf{k}_{T a} d^{2} \mathbf{k}_{T b} \frac{x_{a} x_{b}}{x_{a}-\left(p_{T} / \sqrt{s}\right) e^{y}} \times$

$\times\left[q_{i}\left(x_{a} \mathbf{k}_{T a}\right) \Delta_{N} G\left(x_{b}, \mathbf{k}_{T b}\right) \times \frac{d \widehat{\sigma}}{d \widehat{t}}\left(q_{i} G \rightarrow q_{i} \gamma\right)+\right.$

$\left.+G\left(x_{a}, \mathbf{k}_{T a}\right) \Delta_{N} q_{i}\left(x_{b}, \mathbf{k}_{T b}\right) \frac{d \widehat{\sigma}}{d \widehat{t}}\left(G q_{i} \rightarrow q_{i} \gamma\right)\right]$.

Here, $\sigma^{\uparrow}$ and $\sigma^{\downarrow}$ are the cross-sections of the promptphoton production for the opposite transverse polarizations of one of the colliding protons, $q_{i}\left(x_{a}, \mathbf{k}_{T a}\right) \times$ $\times\left[G\left(x_{a}, \mathbf{k}_{T a}\right)\right]$ is the quark [gluon] distribution function with specified $\mathbf{k}_{T}$, and $\Delta_{N} G\left(x_{b}, \mathbf{k}_{T b}\right) \times$ 


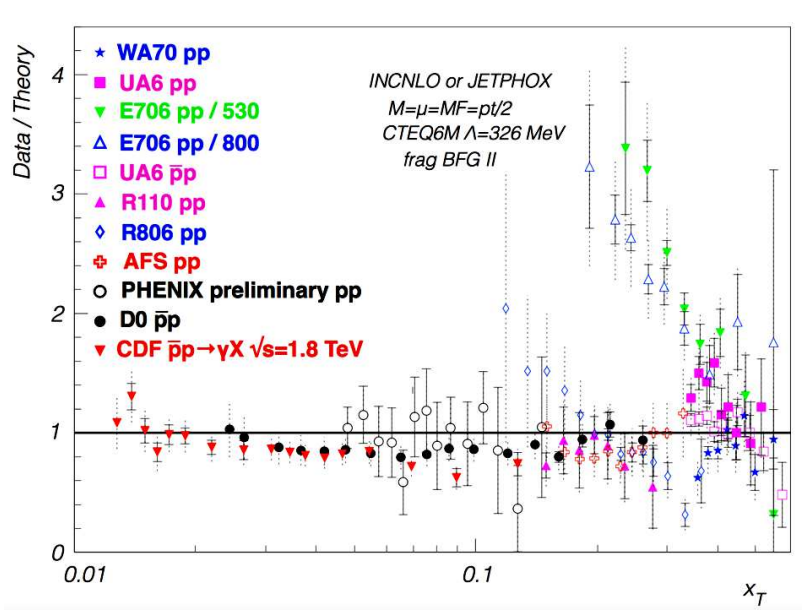

Fig. 1. Measured cross-sections of the prompt-photon production divided by those predicted by theory as a function of $x_{T}[2]$
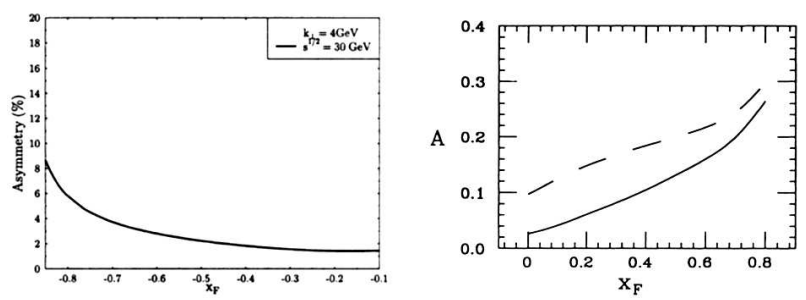

Fig. 2. Theoretical predictions for $A_{\gamma}^{N}$ at $\sqrt{s}=30 \mathrm{GeV}$ and $p_{T}=4 \mathrm{GeV} / c$ for (left) positive [4] and (right) negative [5] values of $x_{F}$

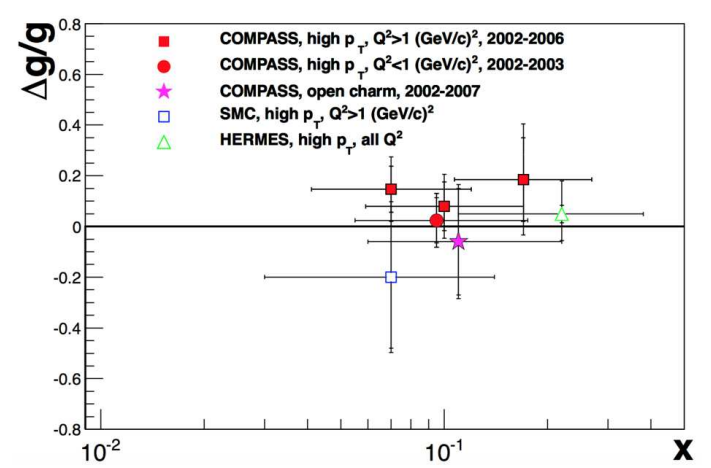

Fig. 3. A compilation of gluon polarization measurements from the open charm and high- $p_{T}$ hadron production [8]

$\times\left[\Delta_{N} q_{i}\left(x_{b}, \mathbf{k}_{T b}\right)\right]$ is the gluon [quark] Sivers function, $\frac{d \widehat{\sigma}}{d \widehat{t}}$ represents the corresponding gluon Compton scattering cross-section. Figure 2 shows theoretical predictions for $A_{\gamma}^{N}$ at $\sqrt{s}=30 \mathrm{GeV}$ and $p_{T}=4 \mathrm{GeV} / c$ for positive [4] (left) and negative [5] (right) values of $x_{F}$. The study of the prompt-photon production at

632 the large transverse momentum with longitudinally polarized proton beams could provide the access to the gluon polarization $\Delta g$ via the measurement of the longitudinal double spin asymmetry $A_{L L}^{\gamma}[6]$. Assuming the dominance of the gluon Compton scattering process, the asymmetry $A_{L L}^{\gamma}$ can be presented as [7]

$$
\begin{aligned}
& A_{L L} \approx \frac{\Delta g\left(x_{a}\right)}{g\left(x_{a}\right)}\left[\frac{\sum_{q} e_{q}^{2}\left[\Delta q\left(x_{b}\right)+\Delta \bar{q}\left(x_{b}\right)\right]}{\sum_{q} e_{q}^{2}\left[q\left(x_{b}\right)+\bar{q}\left(x_{b}\right)\right]}\right] \times \\
& \times \widehat{a}_{L L}(g q \rightarrow \gamma q)+(a \leftrightarrow b) .
\end{aligned}
$$

The second factor in the equation coincides to the lowest order with the spin asymmetry $A_{1}^{p}$ well known from polarized DIS, the partonic asymmetry $\widehat{a}_{L L}$ is calculable in perturbative QCD. Previous results for the gluon polarization show that the gluon polarization is consistent with zero: $|\Delta g / g|< \pm 0.2$ [8], while the $A_{1}^{p}$ asymmetry is about 0.2 for $x \simeq 0.1[9]$.

Thus, under the given experimental conditions, it is possible to gain access to the gluon Sivers function, as well as to the gluon polarization (helicity).

\section{The SPD Detector at NICA}

The study of the gluon structure through the promptphoton production is planned at the SPD experiment at the new accelerator complex NICA (Nuclotronbased Ion Collider fAcility) which is currently under construction at the Joint Institute for Nuclear Research (Dubna, Russia).

The possibility to have high-luminosity collisions (up to $10^{32} \mathrm{~cm}^{-2} \mathrm{~s}^{-1}$ at $\sqrt{s_{p p}}=27 \mathrm{GeV}$ ) of polarized protons and deuterons at the NICA collider allows studying spin- and polarization-dependent effects in hadron collisions.

The current design of the SPD setup foresees three modules: two end-caps and a barrel section. Each part has an individual magnetic system: solenoidal for the end-caps and toroidal for the barrel part of the setup. Main detector systems are the following: Range System (RS) (for muon identification), Electromagnetic calorimeter (ECal), PID/Time-ofFlight system, Main Tracker (TR), and Vertex Detector (VD).

Photons should be detected by the lead-scintillator electromagnetic calorimeter ("shashlyk"-type), which is placed inside the Range System and consists of three parts: the barrel one and two end-caps. Each 
part has a depth of about $12.5 X_{0}$, which is sufficient to fully contain the highly energetic electromagnetic showers considered in this analysis. The energy resolution is planned to be about $5 \% / \sqrt{E[\mathrm{GeV}]}$. The acceptance of the calorimeter in polar angle is between $2^{\circ}$ and $178^{\circ}$.

\section{Prompt Photons at SPD}

The object-oriented $\mathrm{C}++$ toolkit SPDroot based on the FairRoot framework [10] was used for the MonteCarlo simulation of the detector response. The SPD setup description is based on the ROOT geometry while the transportation of secondary particles through a material of the setup, and the simulation of the detector response is provided by the GEANT4 code. The standard multipurpose generators like PYTHIA6, PYTHIA8, FRITIOF could be used for the simulation of primary interactions.

Energy deposition in a connected area in the ECal is called a cluster. If, in the course of extrapolation, the track does not rest against a cluster, such a cluster is considered as neutral, and vice versa. The main issue of the future analysis will be the correct identification of prompt-photon clusters.

The study of background contributions and possibilities of their suppression is almost the main task. On the experience of previous experiments, the main background components are:

- decay photons. Most of them (almost 96\%) are coming from the decays of $\pi^{0}$ and $\eta$ mesons;

- fragmentation photons produced in the process of fragmentation of color partons with large transverse momenta;

- photons produced in the other parts of the facility due to the interaction of particles with a material of the setup;

- neutral hadrons like $n, K^{0}$, etc. and their antiparticles that are identified in the calorimeter as neutral clusters;

- "charged" clusters in the ECal misidentified as "neutral" ones due to the inefficiency of the track finding and reconstruction algorithms;

- the so-called "double" clusters which are a result of the overlapping of showers produced by different particles in ECal. The special case is the clusters produced by energetic $\pi^{0}$ decays into two photons flying at a very small angle relative to each other.

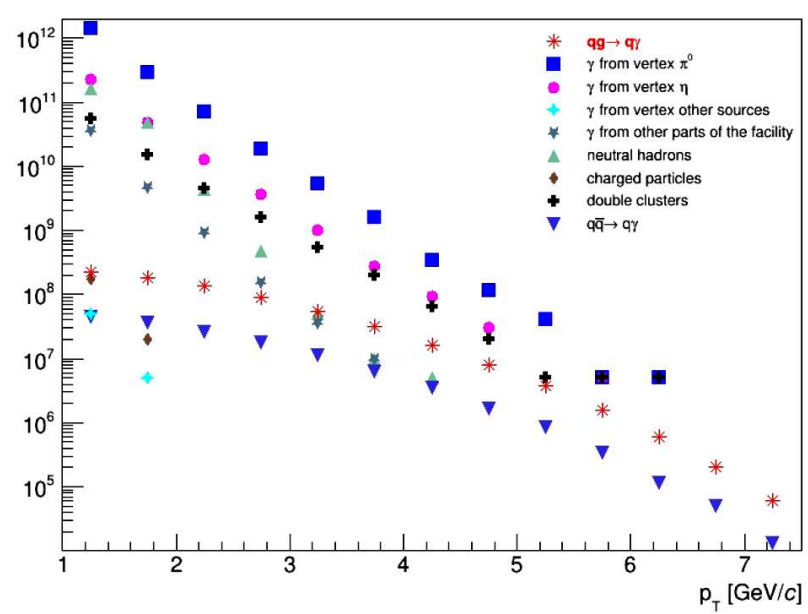

Fig. 4. Contributions of each background component for $p-p$ collisions at $\sqrt{s}=26 \mathrm{GeV}$ shown together with the promptphoton production

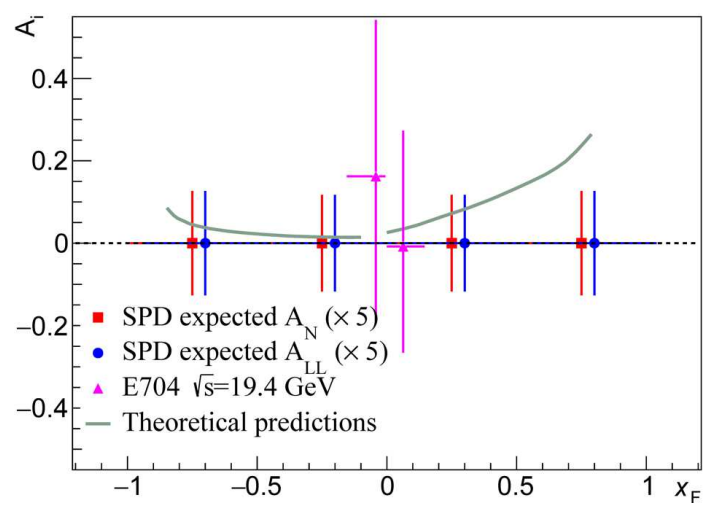

Fig. 5. Expected accuracy of $A_{N}$ and $A_{L L}$ asymmetries with $p_{T}>6 \mathrm{GeV} / c$ as a function of the $x_{F}$-variable

The expected contributions of each background component mentioned above as a function of the transverse momentum $p_{T}$ calculated with the use of the cluster energy and position are shown in Fig. 4 for $p$ - $p$ collisions at $\sqrt{s}=26 \mathrm{GeV}$.

As could be concluded from Fig. 4, the low $p_{T}$ region is useless for any studies of prompt-photons due to a huge background. The signal-to-background ratio at $p_{T}=1 \mathrm{GeV} / c$ is about $10^{-4}$. Only at high values of the transverse momentum, it is possible to separate statistically the signal and the background. A reasonable cut on the transverse momentum of a photon has to be applied in order to maximize the accuracy of the planned measurements.

The background suppression process could be divided into two stages. At first, all photons from the 
reconstructed $2 \gamma$ decays of $\pi^{0}$ and $\eta$ mesons are rejected. After such rejection, the sample still contains an admixture of photons from $2 \gamma$ decays. This residual admixture could be statistically subtracted basing on the Monte-Carlo information about properties of the SPD setup. The subtraction procedure can be illustrated by the following equation:

$\sigma \sim N_{\text {prompt }}=N_{\text {single } \gamma}-N_{\pi^{0}, \eta} \times k$,

where $N_{\text {single } \gamma}$ is a number of candidates to be prompt-photons, $N_{\pi^{0}}$ is a number of reconstructed $2 \gamma$ decays of $\pi^{0}$ and $\eta$, and $k$ is a factor to be determined from the Monte-Carlo procedure. The typical value of the $k$ factor is 0.76 .

To estimate the accuracy of the measurement of asymmetries, the signal and the background MonteCarlo samples were produced. For the simulation of primary $p$ - $p$ collisions with $\sqrt{s}=26 \mathrm{GeV}$, the PYTHIA6 [11] generator with the standard settings was used. The estimation was performed for $10^{7} \mathrm{~s}$ (one year) of data taking with an average luminosity $10^{32} \mathrm{~s}^{-1} \mathrm{~cm}^{-2} .100 \%$ polarization of proton beams was supposed.

Using Eq. (4) and the cut $p_{T}>6 \mathrm{GeV} / c$ which removed most of the background and assuming that the relative accuracy $d k / k=0.02$ could be achieved, the preliminary results on the expected accuracy of the $A_{N}$ and $A_{L L}$ asymmetries measurement in the SPD experiment were obtained. The results for four subranges of $x_{F}$-variable are shown in comparison with the E704 measurements [12] and the theoretical predictions $[4,5]$ in Fig. 5. The expected $A_{N}$ and $A_{L L}$ accuracies are multiplied by a factor of 5 and shown by the error bars in respect to zero values of asymmetries. The uncertainties related to polarization and luminosity measurements are not taken into account.

\section{Conclusions}

The study of the polarized and non-polarized gluon contents of a nucleon is one of the main physical tasks of the planned SPD experiment at the NICA collider. The prompt-photon production via the gluon Compton scattering is the most promising process for that. The precision measurement of the $A_{N}$ and $A_{L L}$ spin asymmetries with transversely and longitudinally polarized proton and deuteron beams provides the access to the gluon Sivers and helicity functions, respectively. The preliminary studies of the background conditions show that the accuracy for the asymmetries of about $2 \%$ could be achieved in the wide range of $x_{F}$.

1. Conceptual and technical design of the Spin Physics Detector (SPD) at the NICA collider. https:// indico.jinr.ru/getFile.py/access?resId $=11 \&$ materialId $=3$ \&confId $=718$ (2018).

2. P. Aurenche, M. Fontannaz, J.-P. Guillet, E. Pilon, M. Werlen. A new critical study of photon production in hadronic collisions. Phys. Rev. D 73, 094007 (2006).

3. I. Schmidt, J. Soffer, J.-J. Yang. Transverse single spin asymmetries in photon production. Phys. Lett. B 612, 258B (2005).

4. N. Hammon, B. Ehrnsperger, A. Schaefer. Single-transverse spin asymmetry in prompt photon production. J. Phys. G 24, 991 (1998).

5. J.-W. Qiu, G.F. Sterman. Single transverse spin asymmetries in direct photon production. Nucl. Phys. B 378, 52 (1992).

6. H.-Y. Cheng, S.-N. Lai. Spin asymmetry in proton proton collisions as a probe of sea and gluon polarization in a proton. Phys. Rev. D 41, 91 (1990).

7. G. Bunce, N. Saito, J. Soffer, W. Vogelsang. Prospects for spin physics at RHIC, Ann. Rev. Nucl. Part. Sci. 50, 525B (2000).

8. C. Adolph, et al. Leading and next-to-leading order gluon polarization in the nucleon and longitudinal double spin asymmetries from open charm muoproduction. Phys. Rev. D 87, 052018 (2013).

9. C. Adolph, et al. The spin structure function $g_{1}^{\mathrm{p}}$ of the proton and a test of the Bjorken Sum Rule, Phys. Rev. B 753, (2015)

10. M. Al-Turany, D. Bertini, R. Bertini, et al. The FairRoot framework, IOP Publishing 396, 022001 (2012).

11. T. Sjostrand, S. Mrenna, P.Z. Skands. PYTHIA 6.4 Physics and Manual. JHEP 0605, 026 (2006).

12. D.L. Adams, et al. Measurement of single spin asymmetry for direct photon production in pp collisions at $200-\mathrm{GeV} / c$. Phys. Lett. B 345, 569 (1995).

Received 08.07.19

\section{А. Римбекова}

ДОСЛІДЖЕННЯ ПОЛЯРИЗОВАНОЇ СТРУКТУРИ ПРОТОНА ЗА ДОПОМОГОЮ ФОТОНАРОДЖЕННЯ В ЕКСПЕРИМЕНТІ SPD НА КОЛАЙДЕРІ NICA

$\mathrm{P}$ е $з$ ю м е

Фотони, утворені в жорсткому розсіянні партонів, так звані миттєві фотони, дають інформацію про внутрішню структуру адронів. Колайдер NICA зможе забезпечити нові дані про народження миттєвих фотонів в неполяризованих та поляризованих процесах фотонарождення, що, в свою чергу, дасть інформацію про спінові функції розподілу глюонів. В даній статті представлено фізику поляризації із миттєвими фотонами і можливості детектора SPD в таких експериментах.

ISSN 2071-0194. Ukr. J. Phys. 2019. Vol. 64, No. 7 